\title{
TITLE:
}

\section{Graph orientations with set connectivity requirements}

AUTHOR(S):

Fukunaga, Takuro

CITATION:

Fukunaga, Takuro. Graph orientations with set connectivity

requirements. Discrete Mathematics 2012, 312(15): 2349-2355

ISSUE DATE:

2012-08

URL:

http://hdl.handle.net/2433/157363

RIGHT:

C 2012 Elsevier B.V.; この論文は出版社版でありません。引用の際には 出版社版をご確認ご利用ください。; This is not the published version. Please cite only the published version. 


\title{
Graph Orientations \\ with Set Connectivity Requirements
}

\author{
Takuro Fukunaga \\ Department of Applied Mathematics and Physics, \\ Graduate School of Informatics, Kyoto University, Kyoto 606-8501, Japan \\ takuro@amp.i.kyoto-u.ac.jp
}

\begin{abstract}
In an undirected or a directed graph, the edge-connectivity between two disjoint vertex sets $X$ and $Y$ is defined as the minimum number of edges or arcs that should be removed for disconnecting all vertices in $Y$ from those in $X$. This paper discusses how to construct a directed graph from a given undirected graph by orienting edges so as to preserve the edge-connectivity on pairs of vertex sets as much as possible. We present several bounds on the gap between the edge-connectivities in the undirected graph and in the obtained directed graphs, which extends the Nash-Williams' strong orientation theorem.

Keywords: edge-connectivity, graph orientation, group Steiner tree packing
\end{abstract}

\section{Introduction}

An orientation $D=(V, A)$ of an undirected graph $G=(V, E)$ is a digraph obtained by replacing each undirected edge $e \in E$ with an arc from one end vertex of $e$ to the other. In an orientation problem, we are asked whether $G$ has an orientation satisfying given connectivity demands. This is a basic problem in combinatorial optimization, and many beautiful results have been produced so far (e.g., $[1,2,3,4,5])$. The main purpose of this paper is to discuss possibility to extend those results by introducing a general concept of the edge-connectivity.

Usually the edge-connectivity is defined on pairs of vertices. On the other hand, this paper deals with the edge-connectivity defined on pairs of vertex sets. Let $X$ and $Y$ be nonempty disjoint subsets of $V$, i.e., $X, Y \in 2^{V}$ and $X \cap Y=\emptyset$. We define the edge-connectivity $\lambda_{G}(X, Y)$ between $X$ and $Y$ in an undirected graph $G=(V, E)$ as $\min \left\{d_{G}(Z) \mid Z \in 2^{V}, X \subseteq\right.$ $Z \subseteq V-Y\}$ where $d_{G}(Z)$ stands for the number of edges joining vertices in $Z$ to those in $V-Z$. Equivalently $\lambda_{G}(X, Y)$ is the edge-connectivity $\lambda_{G^{\prime}}(x, y)$ between two vertices $x$ and $y$ in the graph $G^{\prime}$ obtained from $G$ by shrinking $X$ and $Y$ into single vertices $x$ and $y$, respectively. For a digraph $D$, the arc-connectivity $\lambda_{D}(X, Y)$ from $X$ to $Y$ is defined as $\min \left\{\delta_{D}(Z) \mid Z \in 2^{V}, X \subseteq Z \subseteq V-Y\right\}$ where $\delta_{D}(Z)$ stands for the number of arcs from vertices in $Z$ to those in $V-Z$. $\lambda_{D}(X, Y)$ is also defined as the arc-connectivity $\lambda_{D^{\prime}}(x, y)$ from $x$ to $y$ in the digraph $D^{\prime}$ obtained from $D$ by shrinking $X$ and $Y$ into single vertices $x$ and $y$, respectively.

The connectivity between vertex sets is a useful notion in practice. For example, let $X$ be a set of servers providing the same service in a communication network represented by an undirected graph $G=(V, E)$, and suppose that a vertex $v \in V-X$ represents a client of the 


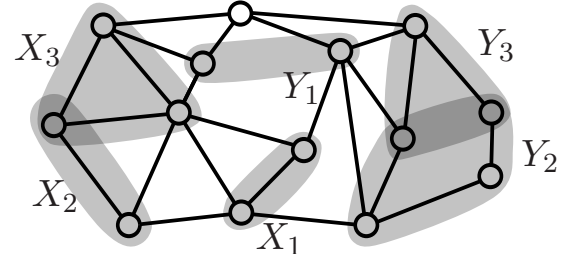

$G$

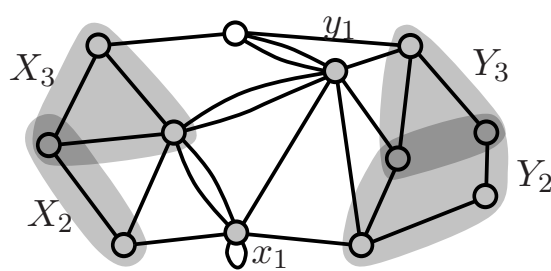

$G^{\prime}$

Figure 1: An undirected graph and pairs of vertex subsets

service. Then $\lambda_{G}(\{v\}, X)$ stands for the minimum number of links which should be broken for disconnecting the client from all servers. By such motivation, several optimization problems defined by the edge-connectivity between vertex sets are considered (e.g., graph augmentation problem $[6,7,8]$, source location problem [9], and minimum cost subgraph problem [10]).

In this paper, we discuss the existence of orientations that satisfy demands defined on given pairs of vertex sets. We ask how large connectivity is necessary for guaranteeing that undirected graphs have such orientations. This is formulated as the following question. Note that $\mathbb{Z}$ denotes the set of integers, and $\mathbb{R}$ denotes the set of reals.

Question 1. Let $G=(V, E)$ be an undirected graph, and $\left\{X_{i}, Y_{i}\right\}$ be pairs of disjoint subsets of $V$ with connectivity demands $f_{i} \in \mathbb{Z}$ for $i \in\{1,2, \ldots, \ell\}$. What is the smallest $C \in \mathbb{R}$ such that each undirected graph $G$ with $\lambda_{G}\left(X_{i}, Y_{i}\right) \geq C f_{i}, i \in\{1,2, \ldots, \ell\}$ has an orientation $D$ with $\min \left\{\lambda_{D}\left(X_{i}, Y_{i}\right), \lambda_{D}\left(Y_{i}, X_{i}\right)\right\} \geq f_{i}, i \in\{1,2, \ldots, \ell\}$ ?

As mentioned in [5], $C \geq 2$ is necessary for satisfying the statement in Question 1 even if the connectivity demands are defined on vertex pairs. With respect to the edge-connectivity between two vertices, Nash-Williams gave the following best possible result.

Theorem 1 (Nash-Williams [5]). Let $f:\left(\begin{array}{l}V \\ 2\end{array}\right) \rightarrow \mathbb{Z}$ be a demand function, where $\left(\begin{array}{l}V \\ 2\end{array}\right)$ denotes the set of unordered pairs of vertices. Every undirected graph $G$ has an orientation $D$ such that $\lambda_{D}(u, v) \geq f(u, v)$ for each $u, v \in V$ if $\lambda_{G}(u, v) \geq 2 f(u, v)$ for each $u, v \in V$.

Question 1 is a natural extension of Theorem 1.

One may consider that an answer to Question 1 can be derived by applying Theorem 1 to the graph obtained by shrinking vertex sets in $\left\{X_{i}, Y_{i} \mid i=1,2, \ldots, \ell\right\}$ into single vertices. We notice that this is not true because of the following two reasons. While we are assuming $X_{i} \cap Y_{i}=\emptyset$ for $i=1,2, \ldots, \ell$ in Question 1 , there are possibly intersecting sets belonging to different pairs, i.e., $Z \cap Z^{\prime} \neq \emptyset$ may hold for some $Z \in\left\{X_{i}, Y_{i}\right\}$ and $Z^{\prime} \in\left\{X_{j}, Y_{j}\right\}$ with $i \neq j$. In addition, even if all sets in the given pairs are disjoint, shrinking a set may change the edge-connectivity of other pairs. For example, see Figure 1 illustrating a graph $G$ with pairs $\left\{X_{1}, Y_{1}\right\},\left\{X_{2}, Y_{2}\right\}$ and $\left\{X_{3}, Y_{3}\right\}$ of subsets of $V$, and $G^{\prime}$ obtained by shrinking $X_{1}$ and $Y_{1}$ into single vertices $x_{1}$ and $y_{1}$. Although the edge-connectivity of $\left\{X_{2}, Y_{2}\right\}$ is not changed by the shrinking $\left(\lambda_{G}\left(X_{2}, Y_{2}\right)=\lambda_{G^{\prime}}\left(X_{2}, Y_{2}\right)=4\right)$, the edge-connectivity of $\left\{X_{3}, Y_{3}\right\}$ is $\left(\lambda_{G}\left(X_{3}, Y_{3}\right)=4\right.$ and $\left.\lambda_{G^{\prime}}\left(X_{3}, Y_{3}\right)=5\right)$.

In this paper, we prove the next theorem, which consists of two upper-bounds on $C$ in Question 1. 
Theorem 2. Let $G=(V, E)$ be an undirected graph, and $\left\{X_{i}, Y_{i}\right\}$ be pairs of disjoint subsets of $V$ with connectivity demands $f_{i} \in \mathbb{Z}$ for $i \in\{1,2, \ldots, \ell\}$. Let $C_{1}=2 \max \left\{\left|X_{i}\right|+\left|Y_{i}\right|-1 \mid\right.$ $i=1,2, \ldots, \ell\}$ and $C_{2}=4 \ell-2$. If an undirected graph $G$ satisfies $\lambda_{G}\left(X_{i}, Y_{i}\right) \geq \min \left\{C_{1}, C_{2}\right\} f_{i}$ for $i \in\{1,2, \ldots, \ell\}$, then $G$ has an orientation $D$ with $\min \left\{\lambda_{D}\left(X_{i}, Y_{i}\right), \lambda_{D}\left(Y_{i}, X_{i}\right)\right\} \geq f_{i}$ for $i \in\{1,2, \ldots, \ell\}$.

We present the proofs for $C_{1}$ in Section 3 and for $C_{2}$ in Section 4. The former bound is derived from an observation presented in [10], and the latter one is proven by applying the theorem due to Frank [1] iteratively. In Section 4, we also show that $C_{2}$ can be improved slightly when an orientation $D$ needs to satisfy $\lambda_{D}\left(X_{i}, Y_{i}\right) \geq f_{i}$ instead of $\min \left\{\lambda_{D}\left(X_{i}, Y_{i}\right), \lambda_{D}\left(Y_{i}, X_{i}\right)\right\} \geq f_{i}$ for $i \in\{1,2, \ldots, \ell\}$. Moreover we give upper- and lowerbounds on the difference between $\min \left\{\lambda_{D}\left(X_{i}, Y_{i}\right), \lambda_{D}\left(Y_{i}, X_{i}\right)\right\}$ and $\lambda_{G}\left(X_{i}, Y_{i}\right)$ in Section 2.

Furthermore, we consider the case where the demand is rooted. Namely $G$ contains a vertex $r \in V$, called root, such that $X_{i}=\{r\}$ for all $i \in\{1,2, \ldots, \ell\}$, and the demand is defined on only the arc-connectivity from the root to $Y_{i}$. In this case, we have the next question similar to Question 1.

Question 2. Let $G=(V, E)$ be an undirected graph, $r \in V, Y_{i} \subseteq V-r$ for $i \in\{1,2, \ldots, \ell\}$, and $f_{i} \in \mathbb{Z}$ for $i \in\{1,2, \ldots, \ell\}$. What is the smallest $C \in \mathbb{R}$ such that each undirected graph $G$ with $\lambda_{G}\left(r, Y_{i}\right) \geq C f_{i}, i \in\{1,2, \ldots, \ell\}$ has an orientation $D$ with $\lambda_{D}\left(r, Y_{i}\right) \geq f_{i}$, $i \in\{1,2, \ldots, \ell\}$ ?

If some $C$ satisfies the statement in Question 1, then it also satisfies the statement in Question 2. Moreover $C=2$ remains best possible for Question 2 even if each $Y_{i}, i \in$ $\{1,2, \ldots, \ell\}$ is singleton. This is implied by an example where $G$ is the union of $k$ copies of a cycle and $|V| \geq 3$. This graph satisfies $\lambda_{G}(r, v)=2 k$ for each $v \in V-r$, but it has no orientation $D$ such that $\lambda_{D}(r, v) \geq k+1$ for all $v \in V-r$. In Section 5, we prove the next theorem, which answers to Question 2.

Theorem 3. Let $G=(V, E)$ be an undirected graph, $r \in V, Y_{i} \subseteq V-r$ for $i \in\{1,2, \ldots, \ell\}$, and $f_{i} \in \mathbb{Z}$ for $i \in\{1,2, \ldots, \ell\}$. If an undirected graph $G$ satisfies $\lambda_{G}\left(r, Y_{i}\right) \geq \ell f_{i}$ for $i \in\{1,2, \ldots, \ell\}$, then $G$ has an orientation $D=(V, A)$ with $\lambda_{D}\left(r, Y_{i}\right) \geq f_{i}$ for $i \in\{1,2, \ldots, \ell\}$.

In the rest of this introduction, let us review the difficulty of our problems from the view point of demand functions. We say that a digraph $D=(V, A)$ covers a demand function $h: 2^{V} \rightarrow \mathbb{Z}$ if $\rho_{D}(X) \geq h(X)$ for all non-empty $X \in 2^{V}$ where $\rho_{D}(X)$ denotes the number of arcs from vertices in $V-X$ to those in $X$.

Subsets $X$ and $Y$ of $V$ are called intersecting if all of $X-Y, Y-X, X \cap Y$ are nonempty. A set function $h: 2^{V} \rightarrow \mathbb{Z}$ is called intersecting $G$-supermodular if $h(X)+h(Y) \leq$ $h(X \cup Y)+h(X \cap Y)+d_{G}(X, Y)$ holds for each intersecting $X, Y \in 2^{V}$ where $d_{G}(X, Y)$ denotes the number of edges in $G$ joining vertices in $X-Y$ and those in $Y-X$. If $h$ satisfies $h(X)+h(Y) \leq h(X \cup Y)+h(X \cap Y)$ for each intersecting $X, Y \in 2^{V}$, then $h$ is called intersecting supermodular. C8 For example, $-\rho_{D}$ is intersecting supermodular (see e.g., [2]).

The following theorem is due to Frank [1].

Theorem 4 (Frank [1]). Let $G$ be an undirected graph and $h$ be an intersecting $G$-supermodular function (with possible negative values). There is an orientation of $G$ covering $h$ if and only if $d_{G}(\mathcal{P}) \geq \sum_{i=1}^{t} h\left(V_{i}\right)$ holds for every subpartition $\mathcal{P}=\left\{V_{1}, V_{2}, \ldots, V_{t}\right\}$ of $V$ where $d_{G}(\mathcal{P})$ denotes the number of edges in $G$ entering at least one member of $\mathcal{P}$. 
This theorem is so general that it includes several known orientation theorems. However our setting is not included by this because demands on the edge-connectivity between vertex sets is not captured by intersecting $G$-supermodular functions. In Section 5, we observe that if Theorem 4 can be extended to skew-supermodular demand functions (defined in Section 5), then $C=2$ satisfies the statement in Question 2 .

\section{Upper- and lower-bounds based on the number of odd- degree vertices}

If $G$ is Eulerian, we can obtain an orientation satisfying the demand in Question 1 with $C=2$ by orienting edges along an Eulerian walk of $G$. By this fact, we can give an upper-bound following the approach taken by Nash-Williams [5] for proving Theorem 1. We let $\tau(G)$ stand for the number of odd-degree vertices in $G$.

Theorem 5. Let $G=(V, E)$ be an undirected graph, and let $\left\{X_{1}, Y_{1}\right\},\left\{X_{2}, Y_{2}\right\}, \ldots,\left\{X_{\ell}, Y_{\ell}\right\}$ be pairs of disjoint subsets of $V$. Then $G$ has an orientation $D$ such that

$$
\min \left\{\lambda_{D}\left(X_{i}, Y_{i}\right), \lambda_{D}\left(Y_{i}, X_{i}\right)\right\} \geq\left\lceil\lambda_{G}\left(X_{i}, Y_{i}\right) / 2\right\rceil-\tau(G) / 2
$$

for each $i \in\{1,2, \ldots, \ell\}$.

Proof. Suppose that $\tau(G)=0$. Then $d_{G}(X)$ is even for all $X \in 2^{V}$. We define $D$ as the digraph obtained by orienting edges in $G$ along an Eulerian walk. Since $D$ satisfies $\rho_{D}(X)=d_{G}(X) / 2$ for all $X \in 2^{V}, \min \left\{\lambda_{D}\left(X_{i}, Y_{i}\right), \lambda_{D}\left(Y_{i}, X_{i}\right)\right\} \geq \lambda_{G}\left(X_{i}, Y_{i}\right) / 2$ for each $i \in\{1,2, \ldots, \ell\}$.

Let us consider the case where $\tau(G)>0$. Augment $G$ by adding a perfect matching $M$ on the odd-degree vertices in $G$. Then the obtained undirected graph $G+M$ is Eulerian (i.e., $\tau(G+M)=0)$. Hence $G+M$ has an orientation $D^{\prime}$ such that $\min \left\{\lambda_{D^{\prime}}\left(X_{i}, Y_{i}\right), \lambda_{D^{\prime}}\left(Y_{i}, X_{i}\right)\right\} \geq$ $\lambda_{G+M}\left(X_{i}, Y_{i}\right) / 2$ for all $i \in\{1,2, \ldots, \ell\}$ as mentioned above. Define $D$ as the digraph obtained by removing arcs corresponding to $M$ from $D^{\prime}$. Then $D$ is an orientation of $G$. Since $|M|=\tau(G) / 2, \lambda_{D}\left(X_{i}, Y_{i}\right) \geq \lambda_{D^{\prime}}\left(X_{i}, Y_{i}\right)-\tau(G) / 2$ and $\lambda_{D}\left(Y_{i}, X_{i}\right) \geq \lambda_{D^{\prime}}\left(X_{i}, Y_{i}\right)-\tau(G) / 2$ for all $i \in\{1,2, \ldots, \ell\}$. Since $G+M$ is Eulerian, every cut has even capacity. It means that $\lambda_{G+M}\left(X_{i}, Y_{i}\right)$ is even, and thus $\lambda_{G+M}\left(X_{i}, Y_{i}\right) \geq 2\left\lceil\lambda_{G}\left(X_{i}, Y_{i}\right) / 2\right\rceil$ holds for all $i \in\{1,2, \ldots, \ell\}$.

From these facts, we can derive

$$
\begin{aligned}
\lambda_{D}\left(X_{i}, Y_{i}\right) \geq \lambda_{D^{\prime}}\left(X_{i}, Y_{i}\right)-\tau(G) / 2 & \\
& \geq \lambda_{G+M}\left(X_{i}, Y_{i}\right) / 2-\tau(G) / 2 \geq\left\lceil\lambda_{G}\left(X_{i}, Y_{i}\right) / 2\right\rceil-\tau(G) / 2
\end{aligned}
$$

and

$$
\begin{aligned}
\lambda_{D}\left(Y_{i}, X_{i}\right) \geq \lambda_{D^{\prime}}\left(Y_{i}, X_{i}\right)-\tau(G) / 2 & \\
& \geq \lambda_{G+M}\left(X_{i}, Y_{i}\right) / 2-\tau(G) / 2 \geq\left\lceil\lambda_{G}\left(X_{i}, Y_{i}\right) / 2\right\rceil-\tau(G) / 2
\end{aligned}
$$

for all $i \in\{1,2, \ldots, \ell\}$.

We also have a negative result for Question 1. 
Theorem 6. Define $\left\{X_{1}, Y_{1}\right\},\left\{X_{2}, Y_{2}\right\}, \ldots,\left\{X_{\ell}, Y_{\ell}\right\}$ as all partitions of $V$ into two nonempty subsets (i.e., $\left\{X_{i} \mid i=1,2, \ldots, \ell\right\}=\left\{X \in 2^{V}|0<| X|<| V \mid / 2\right\}$ and $Y_{i}=V-X_{i}$ ). Then $G$ has no orientation $D$ such that

$$
\min \left\{\lambda_{D}\left(X_{i}, Y_{i}\right), \lambda_{D}\left(Y_{i}, X_{i}\right)\right\}>\lambda_{G}\left(X_{i}, Y_{i}\right) / 2-\tau(G) / 4
$$

for all $i \in\{1,2, \ldots, \ell\}$.

Proof. Suppose that $G$ has an orientation $D$ that satisfies (1) for all $i \in\{1,2, \ldots, \ell\}$. Let us consider the case where at least $\tau(G) / 2$ vertices in $D$ have the in-degrees larger than the out-degrees. Let $X$ denote the set of those vertices in $G$, and $E(X)$ denote the set of edges in $G$ whose both end vertices are in $X$. Then $X$ satisfies

$$
\rho_{D}(X)=\sum_{v \in X} \rho_{D}(v)-E(X) \geq \sum_{v \in X}\left(\delta_{D}(v)+1\right)-E(X) \geq \delta_{D}(X)+\tau(G) / 2 .
$$

On the other hand, $\rho_{D}(X)+\delta_{D}(X)=d_{G}(X)$. By these facts, $\delta_{D}(X) \leq d_{G}(X) / 2-\tau(G) / 4$ holds. Hence we have $\lambda_{D}(X, V-X)=\delta_{D}(X) \leq d_{G}(X) / 2-\tau(G) / 4=\lambda_{G}(X, V-X) / 2-$ $\tau(G) / 4$, a contradiction.

If $D$ has at least $\tau(G) / 2$ vertices having the out-degrees larger than the in-degrees, then consider the digraph $D^{\prime}$ obtained by reversing all arcs in $D$. By applying the above argument to $D^{\prime}$, we have a contradiction also in this case.

From Theorem 6, we can observe that the bound in Theorem 5 is higher than the best possible by at most $\tau(G) / 4$. Theorem 6 also implies that $C<\min \left\{4 /\left(2-\tau(G) / \lambda_{G}\left(X_{i}, Y_{i}\right)\right) \mid\right.$ $1 \leq i \leq \ell\}$ does not satisfy the statement in Question 1 in general.

\section{Proof of the bound $C_{1}$ in Theorem 2}

Fukunaga and Nagamochi [10] gave the following useful relationship between the edge-connectivity between vertices and that between vertex sets.

Lemma 1 (Fukunaga, Nagamochi [10]). Let $\{X, Y\}$ be a pair of disjoint subsets of $V$. If $\lambda_{G}(X, Y) \geq k(|X|+|Y|-1)$, then there exists a pair of vertices $x \in X$ and $y \in Y$ such that $\lambda_{G}(x, y) \geq k$.

From this fact, we can derive the bound $C_{1}$ in Theorem 2 .

Theorem 7. Let $G=(V, E)$ be an undirected graph, and let $\left\{X_{1}, Y_{1}\right\},\left\{X_{2}, Y_{2}\right\}, \ldots,\left\{X_{\ell}, Y_{\ell}\right\}$ be pairs of disjoint subsets of $V$ associated with connectivity demands $f_{1}, f_{2}, \ldots, f_{\ell} \in \mathbb{Z}$. If $G$ satisfies $\lambda_{G}\left(X_{i}, Y_{i}\right) \geq 2\left(\left|X_{i}\right|+\left|Y_{i}\right|-1\right) f_{i}$ for each $i \in\{1,2, \ldots, \ell\}$, then it has an orientation $D$ such that

$$
\min \left\{\lambda_{D}\left(X_{i}, Y_{i}\right), \lambda_{D}\left(Y_{i}, X_{i}\right)\right\} \geq f_{i}
$$

for each $i \in\{1,2, \ldots, \ell\}$.

Proof. Since $\lambda_{G}\left(X_{i}, Y_{i}\right) \geq 2\left(\left|X_{i}\right|+\left|Y_{i}\right|-1\right) f_{i}$ holds, there exists a pair of vertices $x_{i} \in X_{i}$ and $y_{i} \in Y_{i}$ such that $\lambda_{G}\left(x_{i}, y_{i}\right) \geq 2 f_{i}$ by Lemma 1 . The orientation $D$ of $G$ given by Theorem 1 satisfies $\min \left\{\lambda_{D}\left(x_{i}, y_{i}\right), \lambda_{D}\left(y_{i}, x_{i}\right)\right\} \geq f_{i}$ for each $i \in\{1,2, \ldots, \ell\}$. Since $\lambda_{D}\left(X_{i}, Y_{i}\right) \geq$ $\lambda_{D}\left(x_{i}, y_{i}\right)$ and $\lambda_{D}\left(Y_{i}, X_{i}\right) \geq \lambda_{D}\left(y_{i}, x_{i}\right), D$ is a required orientation.

Theorem 7 coincides with Theorem 1 when all demands are defined for vertex pairs. The bound in Theorem 7 is tight in this sense, but this does not deny possibility to improve the bound for the other case. 


\section{Proof of the bound $C_{2}$ in Theorem 2}

In this section, we provide the proof for bound $C_{2}$ in Theorem 2. For this, we need the next lemma.

Lemma 2. Let $G=(V, E)$ be an undirected graph. Moreover let $\left(X_{1}, Y_{1}\right),\left(X_{2}, Y_{2}\right), \ldots,\left(X_{\ell}, Y_{\ell}\right)$ be ordered pairs of disjoint subsets of $V$ associated with connectivity demands $f_{1}, f_{2}, \ldots, f_{\ell} \in \mathbb{Z}$ such that $f_{1} \leq f_{2} \leq \cdots \leq f_{\ell}$. If $G$ satisfies $\lambda_{G}\left(X_{i}, Y_{i}\right) \geq(2 i-1) f_{i}$ for all $i \in\{1,2, \ldots, \ell\}$, then $G$ has an orientation $D$ such that $\lambda_{D}\left(X_{i}, Y_{i}\right) \geq f_{i}$ for all $i \in\{1,2, \ldots, \ell\}$.

Proof. Let $M_{i}=\left(V, E_{i}, A_{i}\right), i \in\{1,2, \ldots, \ell\}$ denote mixed graphs obtained from $G$ by orienting some edges where $E_{i}$ is the set of undirected edges and $A_{i}$ is the set of $\operatorname{arcs}$ in $M_{i}$. We let $G_{i}$ denote the undirected graph $\left(V, E_{i}\right)$, and $D_{i}$ denote the digraph $\left(V, A_{i}\right)$. For proving Lemma 2, we show that it is possible to construct $M_{1}, M_{2}, \ldots, M_{\ell}$ inductively so that $M_{i}$ satisfies

$$
\lambda_{D_{i}}\left(X_{j}, Y_{j}\right) \geq f_{j} \text { for } j \in\{1,2, \ldots, i\},
$$

and

$$
\delta_{D_{i}}(Z) \leq i\left(\rho_{D_{i}}(Z)+f_{i}\right) \text { for } Y_{j} \subseteq Z \subseteq V-X_{j} \text { with } j \in\{i+1, i+2, \ldots, \ell\} .
$$

First, let us show how to construct $M_{1}$. Since $\lambda_{G}\left(X_{1}, Y_{1}\right) \geq f_{1}, G$ contains at least $f_{1}$ edge-disjoint paths between $X_{1}$ and $Y_{1}$. Orient edges in the paths from $X_{1}$ to $Y_{1}$. Then the obtained mixed graph satisfies the conditions (2) and (3) for $i=1$. In fact, the first condition $\lambda_{D_{1}}\left(X_{1}, Y_{1}\right) \geq f_{1}$ holds by the existence of arc-disjoint $f_{1}$ directed paths from $X_{1}$ to $Y_{1}$. The second condition $\delta_{D_{1}}(Z) \leq \rho_{D_{1}}(Z)+f_{1}$ holds because a directed path containing $k$ arcs entering $Z$ can have at most $k$ arcs leaving $Z$ if the path starts at a vertex in $V-Z$, and at most $k+1$ arcs leaving $Z$ otherwise.

Now suppose that we have $M_{i}$ for some $1 \leq i<\ell$. We show how to construct $M_{i+1}$ from $M_{i}$. Let $M^{\prime}=\left(V^{\prime}, E^{\prime}, A^{\prime}\right)$ be the mixed graph obtained from $M_{i}$ by shrinking $X_{i+1}$ into a single vertex $x$, shrinking $Y_{i+1}$ into a single vertex $y$, and deleting generated loops. Define a set function $h: 2^{V^{\prime}} \rightarrow \mathbb{Z}$ so that

$$
h(Z)= \begin{cases}f_{i+1}-\rho_{D^{\prime}}(Z) & \text { if } y \in Z \subseteq V^{\prime}-x, \\ -f_{i+1}-\rho_{D^{\prime}}(Z) & \text { if } x \in Z \subseteq V^{\prime}-y, \\ -\rho_{D^{\prime}}(Z) & \text { otherwise }\end{cases}
$$

Then we have a helpful property of $h$ as follows.

Claim 1. Function $h$ is intersecting supermodular.

Proof. As mentioned in Section 1, $-\rho_{D^{\prime}}$ is intersecting supermodular. Since intersecting supermodularity is closed under addition, it suffices to show that $h^{\prime}:=h+\rho_{D^{\prime}}$ is intersecting supermodular. That is to say, the claim is proven if we show that for intersecting $Z, W \in 2^{V^{\prime}}$ (i.e., all of $Z \cap W, Z-W$ and $W-Z$ are non-empty),

$$
h^{\prime}(Z)+h^{\prime}(W) \leq h^{\prime}(Z \cap W)+h^{\prime}(Z \cup W)
$$

holds. Notice that $h^{\prime}$ returns only three different values. We prove (4) by investigating cases defined according to the values of $h^{\prime}(Z)$ and $h^{\prime}(W)$.

Let $h^{\prime}(Z)=h^{\prime}(W)=f_{i+1}$. In this case, $y \in Z \cap W, Z \cup W$ and $x \notin Z \cap W, Z \cup W$ hold, which implies $h^{\prime}(Z \cap W)=h^{\prime}(Z \cup W)=f_{i+1}$. Hence (4) holds. 
Let $h^{\prime}(Z)=-f_{i+1}$ and $h^{\prime}(W)=f_{i+1}$. In this case, $h^{\prime}(Z \cap W)=0$ because $x, y \notin Y \cap Z$, and $h^{\prime}(Z \cup W)=0$ because $x, y \in Y \cup Z$. Hence (4) holds.

Let $h^{\prime}(Z)=0$ and $h^{\prime}(W)=f_{i+1}$. If $x, y \in Z$, then $x \notin Z \cap W, y \in Z \cap W$ and $x, y \in Z \cup W$ hold, which implies that $h^{\prime}(Z \cap W)=f_{i+1}$ and $h^{\prime}(Z \cup W)=0$. If $x, y \notin Z$, then $x, y \notin Z \cap W$, $x \notin Z \cup W$ and $y \in Z \cup W$ hold, which implies that $h^{\prime}(Z \cap W)=0$ and $h^{\prime}(Z \cup W)=f_{i+1}$. In both cases, (4) holds.

Let $h^{\prime}(Z)=0$ and $h^{\prime}(W)=-f_{i+1}$. If $x, y \in Z$, then $x \in Z \cap W, y \notin Z \cap W$ and $x, y \in Z \cup W$ hold, which implies that $h^{\prime}(Z \cap W)=-f_{i+1}$ and $h^{\prime}(Z \cup W)=0$. If $x, y \notin Z$, then $x, y \notin Z \cap W, x \in Z \cup W$ and $y \notin Z \cup W$ hold, which implies that $h^{\prime}(Z \cap W)=0$ and $h^{\prime}(Z \cup W)=-f_{i+1}$. In both cases, (4) holds.

Let $h^{\prime}(Z)=h^{\prime}(W)=0$. If $x, y \in Z$ and $x, y \in W$, then $x, y \in Z \cap W$ and $x, y \in Z \cup W$, and hence $h^{\prime}(Z \cap W)=h^{\prime}(Z \cup W)=0$ holds. If $x, y \in Z$ and $x, y \notin W$, then $x, y \notin Z \cap W$ and $x, y \in Z \cup W$, and hence $h^{\prime}(Z \cap W)=h^{\prime}(Z \cup W)=0$ holds. If $x, y \notin Z$ and $x, y \notin W$, then $x, y \notin Z \cap W$ and $x, y \notin Z \cup W$, and hence $h^{\prime}(Z \cap W)=h^{\prime}(Z \cup W)=0$ holds. In any cases, (4) holds.

(4) holds when $h^{\prime}(Z)=h^{\prime}(W)=-f_{i+1}$ because $h^{\prime}(Z \cap W) \geq-f_{i+1}$ and $h^{\prime}(Z \cup W) \geq$ $-f_{i+1}$. Therefore the claim is proven.

Recall that intersecting supermodular set functions on $V^{\prime}$ are intersecting $G^{\prime}$-supermodular. Hence we can apply Theorem 4 for obtaining the following fact.

Claim 2. $G^{\prime}$ has an orientation covering $h$.

Proof. We first see that $d_{G^{\prime}}(Z) \geq 2 h(Z)$ holds for any $Z \subseteq V^{\prime}$. It suffices to consider the case where $y \in Z$ and $x \notin Z$ because $h(Z) \leq 0$ in the other case. Notice that $d_{G}(Z)=$ $d_{G^{\prime}}(Z)+\rho_{D^{\prime}}(Z)+\delta_{D^{\prime}}(Z)$. Since $\lambda_{G}\left(X_{i+1}, Y_{i+1}\right) \geq(2 i+1) f_{i+1}, d_{G}(Z) \geq(2 i+1) f_{i+1}$ holds by Menger's theorem. Recall the assumption that $f_{i} \leq f_{i+1}$. By these and condition (3), we have

$$
\begin{aligned}
d_{G^{\prime}}(Z)+(i+1) \rho_{D^{\prime}}(Z) & \geq d_{G^{\prime}}(Z)+\rho_{D^{\prime}}(Z)+\delta_{D^{\prime}}(Z)-i f_{i} \\
& \geq d_{G}(Z)-i f_{i+1} \\
& \geq(i+1) f_{i+1} .
\end{aligned}
$$

Hence $d_{G^{\prime}}(Z) \geq(i+1)\left(f_{i+1}-\rho_{D^{\prime}}(Z)\right)=(i+1) h(Z) \geq 2 h(Z)$.

Let $\mathcal{P}=\left\{V_{1}, V_{2}, \ldots, V_{t}\right\}$ be a subpartition of $V^{\prime}$. It then satisfies $d_{G^{\prime}}(\mathcal{P}) \geq \sum_{j=1}^{t} d_{G^{\prime}}\left(V_{j}\right) / 2 \geq$ $\sum_{j=1}^{t} h\left(V_{j}\right)$. This means that $G^{\prime}$ satisfies the condition presented in Theorem 4 . Therefore $G^{\prime}$ has an orientation covering $h$.

Let $D^{\prime \prime}$ denote the orientation of $G^{\prime}$ covering $h$, and $D^{\prime}+D^{\prime \prime}$ denote the digraph whose arc set consists of the arcs in $D^{\prime}$ and $D^{\prime \prime}$. Then $\lambda_{D^{\prime}+D^{\prime \prime}}(x, y) \geq f_{i+1}$ since each $Z \subseteq V^{\prime}$ with $y \in Z$ and $x \notin Z$ satisfies $\rho_{D^{\prime}+D^{\prime \prime}}(Z)=\rho_{D^{\prime}}(Z)+\rho_{D^{\prime \prime}}(Z) \geq \rho_{D^{\prime}}(Z)+h(Z) \geq f_{i+1}$. Choose $f_{i+1}$ arc-disjoint directed paths from $x$ to $y$ in $D^{\prime}+D^{\prime \prime}$, and call them by $P_{1}, P_{2}, \ldots, P_{f_{i+1}}$. We denote the set of edges both in $E_{i}$ and in $P_{j}$ by $E\left(P_{j}\right)$. Define $M_{i+1}$ as the mixed graph obtained by orienting the edges in $E\left(P_{1}\right) \cup E\left(P_{2}\right) \cup \cdots \cup E\left(P_{f_{i+1}}\right)$ from $x$ to $y$. In the following, we see that the constructed $M_{i+1}$ satisfies conditions (2) and (3).

For each $j \in\{1,2, \ldots, i\}, \lambda_{D_{i+1}}\left(X_{j}, Y_{j}\right) \geq f_{j}$ holds because $A_{i} \subseteq A_{i+1}$ and $M_{i}$ satisfies (2). Moreover, $\lambda_{D_{i+1}}\left(X_{i+1}, Y_{i+1}\right) \geq f_{i+1}$ holds since $D_{i+1}$ contains $f_{i+1}$ arc-disjoint directed paths from $X_{i+1}$ to $Y_{i+1}$. Hence $M_{i+1}$ satisfies (2). 
Let $Z \in 2^{V}$ such that $Y_{j} \subseteq Z \subseteq V-X_{j}$ for some $j \in\{i+2, i+3, \ldots, \ell\}$. Notice that each arc entering or leaving $Z$ in $A_{i+1}-A_{i}$ is part of a directed path from $x$ to $y$ in $D^{\prime \prime}$. Hence $\delta_{D_{i+1}}(Z)-\delta_{D_{i}}(Z) \leq \rho_{D_{i+1}}(Z)+f_{i+1}$ holds. By this fact and the assumption that $M_{i}$ satisfies (3), it holds that

$$
\begin{aligned}
\delta_{D_{i+1}}(Z) & =\delta_{D_{i+1}}(Z)-\delta_{D_{i}}(Z)+\delta_{D_{i}}(Z) \\
& \leq \rho_{D_{i+1}}(Z)+f_{i+1}+i\left(\rho_{D_{i}}(Z)+f_{i}\right) \\
& \leq(i+1)\left(\rho_{D_{i+1}}(Z)+f_{i+1}\right)
\end{aligned}
$$

where the last inequality follows from the properties $\rho_{D_{i}}(Z) \leq \rho_{D_{i+1}}(Z)$ and $f_{i} \leq f_{i+1}$. Therefore $M_{i+1}$ satisfies (3). This completes the proof of Lemma 2 .

The result on $C_{2}$ in Theorem 2 is obtained as follows.

Theorem 8. Let $G=(V, E)$ be an undirected graph, and $\left\{X_{i}, Y_{i}\right\}$ be pairs of disjoint subsets of $V$ with connectivity demands $f_{i} \in \mathbb{Z}$ for $i \in\{1,2, \ldots, \ell\}$. If an undirected graph $G$ satisfies $\lambda_{G}\left(X_{i}, Y_{i}\right) \geq(4 \ell-2) f_{i}$ for $i \in\{1,2, \ldots, \ell\}$, then $G$ has an orientation $D$ with $\min \left\{\lambda_{D}\left(X_{i}, Y_{i}\right), \lambda_{D}\left(Y_{i}, X_{i}\right)\right\} \geq f_{i}$ for $i \in\{1,2, \ldots, \ell\}$.

Proof. The theorem can be proven by applying Lemma 2 for the set of ordered pairs $\left(X_{i}, Y_{i}\right)$ and $\left(Y_{i}, X_{i}\right), i=1,2, \ldots, \ell$.

In addition, we would like to note that Lemma 2 gives a theorem on a slightly weaker connectivity demand.

Theorem 9. Let $G=(V, E)$ be an undirected graph, and $\left(X_{i}, Y_{i}\right)$ be ordered pairs of disjoint subsets of $V$ with connectivity demands $f_{i} \in \mathbb{Z}$ for $i \in\{1,2, \ldots, \ell\}$. If an undirected graph $G$ satisfies $\lambda_{G}\left(X_{i}, Y_{i}\right) \geq(2 \ell-1) f_{i}$ for $i \in\{1,2, \ldots, \ell\}$, then $G$ has an orientation $D$ with $\lambda_{D}\left(X_{i}, Y_{i}\right) \geq f_{i}$ for $i \in\{1,2, \ldots, \ell\}$.

\section{$5 \quad$ Proof of Theorem 3}

In this section, we discuss the case where the connectivity demands are defined on pairs containing the root. Theorem 3 is an immediate consequence of the following lemma.

Lemma 3. Let $r \in V$ and $Y_{1}, Y_{2}, \ldots, Y_{\ell} \subseteq V-r$. An undirected graph $G=(V, E)$ has an orientation $D$ such that $\lambda_{D}\left(r, Y_{i}\right) \geq f_{i}$ for all $i \in\{1,2, \ldots, \ell\}$ if $G$ satisfies $\lambda_{G}\left(r, Y_{i}\right) \geq i f_{i}$ for all $i \in\{1,2, \ldots, \ell\}$.

Proof. Most part of the proof of Lemma 3 is same with the proof of Lemma 2. In Lemma 3, we can show that it is possible to construct mixed graphs $M_{1}, M_{2}, \ldots, M_{\ell}$ such that $M_{i}$ satisfies (2) and

$$
\delta_{D_{i}}(Z) \leq i \rho_{D_{i}}(Z) \text { for } Y_{j} \subseteq Z \subseteq V-r \text { with } j \in\{i+1, i+2, \ldots, \ell\},
$$

which improves (3). Here we mention only the different part from the proof of Lemma 2.

We consider constructing $M_{i+1}$ from $M_{i}$ satisfying (2) and (5). Define $M^{\prime}=\left(V^{\prime}, E^{\prime}, A^{\prime}\right)$ as the mixed graph obtained from $M_{i}$ by shrinking $Y_{i+1}$ into a single vertex $y$. Define a set function $h: 2^{V^{\prime}} \rightarrow \mathbb{Z}$ as in the proof of Lemma 2 by replacing $x$ with $r$. 
Let $G^{\prime}=\left(V^{\prime}, E^{\prime}\right)$ and $D^{\prime}=\left(V^{\prime}, A^{\prime}\right)$. If $y \in Z \subseteq V^{\prime}-r$, then $d_{G^{\prime}}(Z)+\rho_{D^{\prime}}(Z)+\delta_{D^{\prime}}(Z)=$ $d_{G}(Z) \geq \lambda_{G}\left(r, Y_{i+1}\right) \geq(i+1) f_{i+1}$ holds. This and (5) imply

$$
d_{G^{\prime}}(Z)+(i+1) \rho_{D^{\prime}}(Z) \geq d_{G^{\prime}}(Z)+\rho_{D^{\prime}}(Z)+\delta_{D^{\prime}}(Z) \geq(i+1) f_{i+1},
$$

and hence $G^{\prime}=\left(V^{\prime}, E^{\prime}\right)$ satisfies $d_{G^{\prime}}(Z) \geq(i+1) h(Z) \geq 2 h(Z)$ for any $Z$ such that $y \in Z \subseteq$ $V^{\prime}-r$. For the other $Z \in 2^{V^{\prime}}, d_{G^{\prime}}(Z) \geq 2 h(Z)$ holds because $h(Z) \leq 0$. Consequently we can see that $G^{\prime}$ has an orientation covering $h$ as in Claim 2.

From the orientation of $G^{\prime}$ covering $h$, define $M_{i+1}$ in the same way with the proof of Lemma 2. The existence of $f_{i+1}$ arc-disjoint directed paths from $r$ to $Y_{i+1}$ means that $M_{i+1}$ satisfies (2). (5) is also satisfied by $M_{i+1}$ because each of the paths starts at $r \notin Z$.

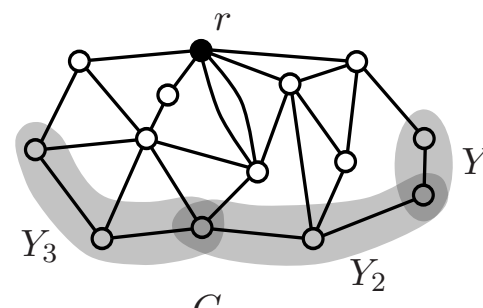

$G$

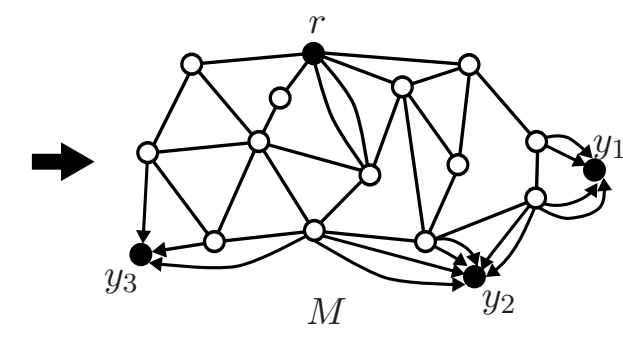

$M$

Figure 2: Construction of $M$ from $G$

In the remainder of this section, we show that the orientation problem with arc-connectivity demands from $r$ to $Y_{i}, i \in\{1,2, \ldots, \ell\}$ can be reduced to the orientation problem in mixed graphs with connectivity demands from $r$ to single vertices. Given graph $G=(V, E)$ with $r \in V$ and $Y_{1}, Y_{2}, \ldots, Y_{\ell} \subseteq V-r$, augment $G$ with a new vertex $y_{i}$ for each $i \in\{1,2, \ldots, \ell\}$ and $f_{i}$ parallel arcs from each $v \in Y_{i}$ to $y_{i}$. Let $M$ denote the obtained mixed graph (see Figure 2). If we can orient the undirected edges in $M$ so that the resultant digraph $D$ satisfies $\lambda_{D}\left(r, y_{i}\right) \geq f_{i}$ for $i \in\{1,2, \ldots, \ell\}$, then it gives an orientation $D^{\prime}$ of $G$ such that $\lambda_{D^{\prime}}\left(r, Y_{i}\right) \geq f_{i}$ for $i \in\{1,2, \ldots, \ell\}$.

Unfortunately we do not know how to solve this orientation problem. If the connectivity demand is defined from $r$ to $v$ for all $v \in V-r$, then Theorem 4 gives a necessary and sufficient condition. However, in the above reduced problem, the connectivity demand is defined only from $r$ to $y_{i}, i \in\{1,2, \ldots, \ell\}$. This demand can not be formulated by $G$ supermodular functions, but by skew supermodular functions. If Theorem 4 can be extended to skew supermodular functions, then it implies that $C=2$ is an answer to Question 2.

\section{Concluding Remarks}

As a concluding remark, let us mention a relationship between rooted $k$-arc-connectivity and tree packings.

For an undirected graph $G=(V, E)$ with a root $r \in V$ and subsets $Y_{i}, i \in\{1,2, \ldots, \ell\}$ of $V-\{r\}$, a group Steiner tree is defined as a tree $T$ in $G$ spanning $r$ and at least one vertex in $Y_{i}$ for each $i \in\{1,2, \ldots, \ell\}$. The packing number of group Steiner trees is defined as the maximum number of edge-disjoint group Steiner trees contained by $G$. Notice that if the packing number is at least $k$, then $G$ has obviously an orientation $D$ that satisfies $\lambda\left(r, Y_{i}\right) \geq k$ for all $i \in\{1,2, \ldots, \ell\}$. We do not know whether its converse holds or not. 
In [10], Fukunaga and Nagamochi have shown that the packing number is at least $k$ if $G$ satisfies $\lambda_{G}\left(r, Y_{i}\right) \geq 2 k\left|Y_{i}\right|$ for all $i \in\{1,2, \ldots, \ell\}$. Based on this observation, they have presented an approximation algorithm for the minimum group Steiner tree problem, which is the problem of finding a minimum cost group Steiner tree ${ }^{1}$. The approximation factor of their algorithm is the gap between the edge-connectivity in $G$ and the packing number. Hence it is important to improve this gap.

Notice that the gap presented by Fukunaga and Nagamochi [10] coincides with Theorem 7. A natural question is whether Theorem 3 can be strengthened to obtain another gap between the edge-connectivity in $G$ and the packing number. This question is formulated as the following conjecture.

Conjecture 1. Let $G=(V, E)$ be an undirected graph with a root $r \in V$ and subsets $Y_{1}, Y_{2}, \ldots, Y_{\ell} \subseteq V-r$. If $\lambda_{G}\left(r, Y_{i}\right) \geq \ell k$ for all $i \in\{1,2, \ldots, \ell\}$, then $G$ contains $k$ edgedisjoint group Steiner trees.

This conjecture implies an $\ell$-approximation algorithm for the group Steiner tree problem. Since the union of minimum cost paths from $r$ to $Y_{i}$ for $i \in\{1,2, \ldots, \ell\}$ is an $\ell$-approximate solution, this implication is not interesting. Nevertheless we believe that the packing of group Steiner trees itself deserves attention.

\section{Acknowledgments}

A preliminary version of this paper appeared in proceedings of the 20th International Symposium on Algorithms and Computation (ISAAC 2009). This work was partially supported by Grant-in-Aid for Scientific Research from the Ministry of Education, Culture, Sports, Science and Technology of Japan.

\section{References}

[1] A. Frank, Orientations of graphs and submodular flows, Conguressus Numerantium 113 (1996) 111-142.

[2] A. Frank, Connections in Combinatorial Optimization, Oxford University Press, 2011.

[3] T. Jordán, On the existence of $k$ edge-disjoint 2-connected spanning subgraphs, Journal of Combinatorial Theory, Series B 95 (2005) 257-262.

[4] T. Király, L.C. Lau, Approximate min! =max theorems for Steiner rooted-orientations of graphs and hypergraphs, Journal of Combinatorial Theory, Series B 98 (2008) 1233-1252.

[5] C. S. J. A. Nash-Williams, On orientations, connectivity and odd vertex pairings in finite graphs, Canadian Journal of Mathematics 12 (1960) 555-567.

[6] T. Ishii, M. Hagiwara, Minimum augmentation of local edge-connectivity between vertices and vertex subsets in undirected graphs, Discrete Applied Mathematics 154 (2006) 23072329.

\footnotetext{
${ }^{1}$ Fukunaga and Nagamochi [10] have actually solved a problem including the group Steiner tree problem by the above-mentioned approach.
} 
[7] T. Ishii, K. Makino, Augmenting edge-connectivity between vertex subsets, in: Proceedings of Computing Theory: The Australian Theory Symposium, 2009, pp. 45-51.

[8] H. Ito, Node-to-area connectivity of graphs, Transactions of the Institute of Electrical Engineers of Japan 11C (1994) 463-469.

[9] M. Bárász, J. Beckder, A. Frank, An algorithm for source location in directed graphs, Operations Research Letters 33 (2005) 221-230.

[10] T. Fukunaga, H. Nagamochi, The set connector problem in graphs, in: Proceedings of the 12th Conference on Integer Programming and Combinatorial Optimization, Vol. 4513 of Lecture Notes in Computer Science, 2007, pp. 484-498. 